\title{
BMJ Open Protocol for a multicentre prehospital randomised controlled trial investigating tranexamic acid in severe trauma: the PATCH-Trauma trial
}

\author{
Biswadev Mitra (D) , ${ }^{1,2,3}$ Stephen Bernard, ${ }^{3,4,5}$ Dashiell Gantner, ${ }^{3,4,6}$ Brian Burns, ${ }^{7,8}$ \\ Michael C Reade (D) , 9,10,11 Lynnette Murray, ${ }^{3,4,6}$ Tony Trapani, ${ }^{3,4,6}$ Veronica Pitt, ${ }^{3}$ \\ Colin McArthur, ${ }^{12}$ Andrew Forbes, ${ }^{3}$ Marc Maegele, ${ }^{13,14}$ Russell L Gruen, ${ }^{15}$ PATCH- \\ Trauma study investigators
}

To cite: Mitra B, Bernard S, Gantner D, et al. Protocol for a multicentre prehospital randomised controlled trial investigating tranexamic acid in severe trauma: the PATCH-Trauma trial. BMJ Open 2021;11:e046522. doi:10.1136/ bmjopen-2020-046522

- Prepublication history is published online only. To view, please visit the journal online (http://dx.doi.org/10.1136/ bmjopen-2020-046522).

Received 02 November 2020 Revised 23 December 2020 Accepted 08 February 2021

Check for updates

(C) Author(s) (or their employer(s)) 2021. Re-use permitted under CC BY-NC. No commercial re-use. See rights and permissions. Published by BMJ.

For numbered affiliations see end of article.

Correspondence to Professor Biswadev Mitra; biswadev.mitra@monash.edu

\section{ABSTRACT}

Introduction Haemorrhage causes most preventable prehospital trauma deaths and about a third of in-hospital trauma deaths. Tranexamic acid (TXA), administered soon after hospital arrival in certain trauma systems, is an effective therapy in preventing or managing acute traumatic coagulopathy. However, delayed administration of TXA appears to be ineffective or harmful. The effectiveness of prehospital TXA, incidence of thrombotic complications, benefit versus risk in advanced trauma systems and the mechanism of benefit remain uncertain. Methods and analysis The Pre-hospital Anti-fibrinolytics for Traumatic Coagulopathy and Haemorrhage (The PATCHTrauma study) is comparing TXA, initiated prehospital and continued in hospital over 8 hours, with placebo in patients with severe trauma at risk of acute traumatic coagulopathy. We present the trial protocol and an overview of the statistical analysis plan. There will be 1316 patients recruited by prehospital clinicians in Australia, New Zealand and Germany. The primary outcome will be the eight-level Glasgow Outcome Scale Extended (GOSE) at 6 months after injury, dichotomised to favourable (GOSE 5-8) and unfavourable (GOSE 1-4) outcomes, analysed using an intention-to-treat (ITT) approach. Secondary outcomes will include mortality at hospital discharge and at 6 months, blood product usage, quality of life and the incidence of predefined adverse events.

Ethics and dissemination The study was approved by The Alfred Hospital Research and Ethics Committee in Victoria and also approved in New South Wales, Queensland, South Australia, Tasmania and the Northern Territory. In New Zealand, Northern A Health and Disability Ethics Committee provided approval. In Germany, Witten/ Herdecke University has provided ethics approval. The PATCH-Trauma study aims to provide definitive evidence of the effectiveness of prehospital TXA, when used in conjunction with current advanced trauma care, in improving outcomes after severe injury.

Trial registration number NCT02187120.

\section{INTRODUCTION}

Every year, over 5 million people die from injury worldwide. ${ }^{1}$ In Australia, injuries result
Strengths and limitations of this study

- A double-blinded randomised controlled design will minimise bias of the results.

- Delivery of the initial study drug in the prehospital phase of trauma care will provide level I evidence on prehospital use of tranexamic acid for trauma.

- The primary outcome is patient-centric being favourable functional status at 6 months after injury.

- Prespecified secondary outcome measures are designed to investigate potential mechanism of actions of tranexamic acid in injured patients.

- The study is enrolling patients from Australia, New Zealand and Germany and results may not be generalisable to all trauma systems.

in approximately 2500 deaths per year, 5000 survivors who are severely disabled and 25000 survivors who bear other long-term disabilities. ${ }^{2}$ Acute haemorrhage is directly responsible for most preventable prehospital trauma deaths and about a third of in-hospital trauma deaths. ${ }^{3}$ Haemorrhage and its management, often involving massive blood transfusion, also contribute to multiorgan failure leading to later mortality and morbidity. ${ }^{45}$

Normal circulatory homeostasis, ensuring both tissue perfusion and rapid plugging of damaged vessels to minimise bleeding, depends on a complex system of concurrent clot formation and clot breakdown (fibrinolysis). Following severe haemorrhage and tissue damage, many patients develop pathological hyperfibrinolysis and a measurable acute traumatic coagulopathy (ATC). Patients with ATC are up to eight times more likely to die within the first 24 hours than trauma patients without coagulopathy. ${ }^{67}$ ATC on admission is also associated with a higher risk of development of acute renal injury, 
multiple organ failure, fewer ventilator-free days and longer stay in the intensive care unit (ICU) and hospital. ${ }^{8}$

Hyperfibrinolysis is the consequence of raised levels of tissue-type plasminogen activator (t-PA) causing excessive plasminogen activation of the fibrin surface and subsequent fibrin dissolution. Mechanistically, plasminogen binds to exposed lysine residues located on the fibrin surface. Once bound to fibrin, plasminogen partially unfolds becoming more accessible to t-PA allowing plasmin to be generated. Tranexamic acid (TXA) is a lysine analogue that competitively inhibits the binding of plasminogen to fibrin, thereby sparing fibrin from plasmin-mediated fibrinolysis. ${ }^{9}$ Many studies in elective surgery have demonstrated TXA reduces blood transfusion requirements. ${ }^{10}$ The most significant study of TXA in trauma care was the Clinical Randomisation of an Antifibrinolytic in Significant Haemorrhage-2 (CRASH-2) trial, which measured the effects of TXA administered in hospital on death, vaso-occlusive events and the receipt of blood transfusion in trauma patients at risk of significant haemorrhage. ${ }^{11}$ The trial found that TXA was associated with significantly reduced deaths due to bleeding and allcause mortality (risk ratio (RR) $0.91 ; 95 \%$ CI 0.85 to 0.97 ; $\mathrm{p}=0.0035)$, an effect that varied inversely with time to treatment. The researchers further found that the beneficial effects were seen when TXA was administered within 3 hours of injury, with potential for harm when administered after 3 hours. ${ }^{12}$

CRASH-2 was the largest randomised controlled trial enrolling patients in the early stages of trauma resuscitation, and its findings have influenced trauma care worldwide. It has also engendered considerable debate, however, and the following issues have prompted calls for further trials prior to indiscriminate application of this therapy in advanced trauma systems. ${ }^{13-20}$

\section{Timing of intervention}

The interpretation of the effects of TXA was complicated by an apparent increase in the risk of death due to bleeding if TXA was administered 3 hours or more following injury. ${ }^{21}$ More detailed analysis of the timing of treatment suggested that while TXA was administered after hospital arrival, there appeared to be homogeneous improvement in outcomes with early administration of TXA. ${ }^{22}$ This suggests that prehospital therapy may be of benefit, but this hypothesis has not yet been adequately explored. $^{23}$

\section{Generalisability}

Almost all patients in CRASH-2 were in low-income and middle-income countries where prehospital care was limited, blood components were uncommonly used and where injury mortality was high. ${ }^{24}$ Seventy-four per cent of the CRASH-2 patients were enrolled in Columbia, Ecuador, Georgia, Nigeria, Egypt and India; only 340 (1.7\%) patients were from Australia, New Zealand, the USA, Canada, Western Europe or the UK, where trauma system improvements have greatly reduced injury mortality and improved functional recovery. Subgroup analyses of CRASH-2 have not addressed this limitation. ${ }^{21} 2225$ In regions with advanced trauma care systems, where preventable trauma deaths have been reduced through other means, it is unclear whether the same riskbenefit ratio of TXA applies. If the number of trauma deaths that could be prevented by use of TXA is fewer, it is possible that the incidence of adverse effects (such as vascular occlusive events) will unfavourably shift the balance of benefit and harm. ${ }^{26}$ Furthermore, it is uncertain whether TXA administered in the prehospital setting alongside current advanced prehospital and in-hospital trauma care including routine blood product use adds additional benefit. ${ }^{27} 28$

\section{Effect size}

CRASH-2 enrolled patients if they were 'at risk of significant haemorrhage', defined as a systolic blood pressure $<90 \mathrm{~mm} \mathrm{Hg}$ or heart rate $>110 /$ min or both, or at clinician discretion. These measures are neither sensitive nor specific for haemorrhage or coagulopathy. It is possible these criteria decreased the proportion of patients who had hyperfibrinolysis and who could have benefitted from TXA, while exposing patients without ATC or significant bleeding to potential procoagulant harm.

\section{Assessment of potential adverse events}

A potential concern relates to altering the delicate balance between thrombogenic and thrombolytic mechanisms in favour of systemic thrombosis, resulting in venous or arterial thromboembolism, ischaemic heart disease or stroke. In CRASH-2, patients receiving TXA were not diagnosed with such vascular occlusive events more often than those who received placebo, and the overall incidence was lower than observed in other trauma cohorts. ${ }^{29}$ This is at odds with a number of observational studies, in which TXA administration seemed to be an independent risk factor for venous thromboembolism, casting doubt on this finding. ${ }^{2030}{ }^{31}$ Seizures, a known risk of high-dose TXA, did not seem to be a significant problem in the CRASH-2 study. ${ }^{32}$

\section{Mechanism of benefit}

An understanding of the mechanisms by which TXA may improve outcomes could assist in tailoring therapy to those who may benefit, while identifying subgroups at risk of adverse events. Plasmin has roles unrelated to fibrinolysis (probably influencing inflammation, immunity, neurological function and neuropathic pain) that may be blocked by TXA. ${ }^{33}$ However, there was no detailed analysis of the immunomodulatory or haemostatic effects of TXA in CRASH-2. Indeed, the mechanism of mortality benefit observed in CRASH-2 was unclear, as there was no difference in transfusion requirements between TXA and placebo groups, and neither sepsis nor neurological outcomes were specifically reported. 
Box 1 Inclusion and exclusion criteria

\section{Inclusion criteria}

- Adult patients (aged $\geq 18$ years).

- Injury through any mechanism.

- Coagulopathy of Severe Trauma score $\geq 3$.

- First dose of study drug can be administered within 3 hours of injury.

- Patients to be transported to a participating trauma centre.

\section{Exclusion criteria}

- Suspected pregnancy.

Nursing home residents.

Age $<18$ years.

\section{METHODS AND ANALYSIS \\ Design}

PATCH-Trauma is an international, multicentre, doubleblind, randomised, placebo-controlled trial that aims to determine the benefits and harms of initiating TXA treatment in the prehospital setting for severely injured patients at high risk of developing ATC.

\section{Participants}

Injured adult patients being transported by ambulance to major trauma services in three countries (Australia, New Zealand and Germany) are eligible for inclusion if assessed as being at high risk of ATC and if the first dose of study drug can be administered within 3 hours of injury. Details of eligibility are listed in box 1 .

\section{COAST assessment}

The validated, five-item Coagulopathy of Severe Trauma (COAST) score is used to assess whether each patient is at high risk of ATC (table 1) ${ }^{34-36}$ COAST is a score that can be easily and rapidly applied in the field by trained paramedics. Patients with COAST scores $\geq 3$ are eligible for enrolment. Patients may be assessed for eligibility at any time in the prehospital setting.

\section{Randomisation and blinding}

Trial packs are prepared by an independent pharmaceutical packaging company (PCI Pharma Services for Australia and New Zealand, Pharmacy University of Nuremberg Erlangen for Germany) with either TXA or placebo using a computer-generated sequence provided by a statistician at Monash University, Department of Epidemiology and Preventive Medicine. Packs are consecutively numbered, opaque, foil parcels with a tamper proof seal. Randomisation sequence is stratified for each state and country participating in the study. The pleiotropic activity of TXA, separate to its antifibrinolytic effect, may have disproportionate effects on patients with traumatic brain injury (TBI) ${ }^{34}$ Therefore, patients are additionally being stratified by the presence of severe TBI defined by a Glasgow Coma Scale (GCS) $<9$ at the time of randomisation. All trial personnel, including the follow-up assessors and participants, are blinded to treatment allocation. Unblinding of the treatment assignment can only occur in the unlikely event of an emergency in which the appropriate treatment of the patient requires knowledge of the study drug.

\section{Study interventions}

Two $10 \mathrm{~mL}$ ampoules containing either $1000 \mathrm{mg}$ TXA or $0.9 \%$ sodium chloride $(\mathrm{NaCl})$ are in each trial pack, labelled with a unique study ID number. The attending clinicians deliver one dose of the trial drug intravenously to the patient as a bolus (over $10 \mathrm{~min}$ ) as soon as practicable after initial assessment of the patient, and before the patient reaches hospital. On arrival to the emergency department of participating hospitals, the second $10 \mathrm{~mL}$ ampoule in the trial pack is added to $1 \mathrm{~L}$ of $0.9 \% \mathrm{NaCl}$ and infused over 8 hours (figure 1). Study medications are ceased permanently for any serious adverse event such as seizure, cardiac arrest or anaphylaxis, and also in the setting of an exclusion criterion being discovered, for example, positive urine or blood pregnancy test or when a 'not for active treatment' directive becomes apparent

\begin{tabular}{|c|c|c|c|}
\hline COAST score variable & Assessment & Result & Score \\
\hline Entrapment (ie, in vehicle) & $\begin{array}{l}\text { Extraction of patient from vehicle or scene of injury requires use } \\
\text { of cutting or lifting devices }\end{array}$ & Yes & 1 \\
\hline \multirow[t]{2}{*}{ Systolic blood pressure (mm Hg) } & Sphygmomanometer & $<100$ & 1 \\
\hline & & $<90$ & 2 \\
\hline & & $<32$ & 2 \\
\hline $\begin{array}{l}\text { Major chest injury likely to require } \\
\text { intervention } \\
\text { (eg, decompression, chest tube) }\end{array}$ & $\begin{array}{l}\text { In the opinion of prehospital clinician, there is likely chest } \\
\text { injury sufficient to require a thoracostomy for pneumothorax or } \\
\text { haemothorax }\end{array}$ & Yes & 1 \\
\hline Likely intra-abdominal or pelvic injury & $\begin{array}{l}\text { In the opinion of the prehospital clinician, there is likely to be } \\
\text { injury to abdominal organs or to the pelvis }\end{array}$ & Yes & 1 \\
\hline
\end{tabular}




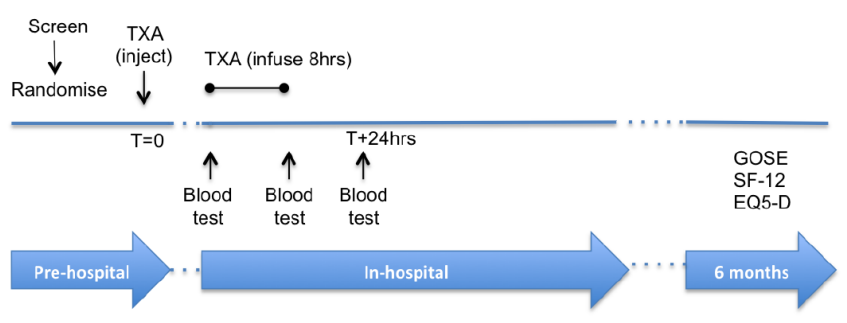

Figure 1 Schema of enrolment and assessment of outcomes. EQ-5D, EuroQol 5-Dimension; GOSE, Glasgow Outcome Scale Extended; SF-12, 12-item Short Form Survey; TXA, tranexamic acid.

or the participant declines further participation in the study. Ultimately, the patient and treating clinician have the right to decide whether to discontinue treatment.

\section{Concomitant care}

Initial assessment and treatment of seriously injured patients follows usual practice. Prehospital clinicians attending the scene continue to resuscitate patients as per their usual ambulance service guidelines. Following arrival at hospital, standard procedures for trauma reception and resuscitation are followed. Specifically, while the trial investigators, including those at each participating site, have confirmed equipoise about the benefit and harms of TXA in these patients and settings, open-label administration of TXA is allowed at clinician discretion, and data on such administration are collected for analysis.

\section{Outcomes}

Details of all outcome measures are listed in box 2. A purpose-built website with an electronic case report form is used for data collection at participating sites. All data are collected by trained research site staff directly from clinical source data. Trained assessors, blinded to the intervention, also collect data on the primary outcome measure at 6 months after injury. A study monitor from the Australian and New Zealand Research Centre, Monash University undertakes site visits and remote checks for study compliance, accuracy and completion of data collection.

Functional recovery (the primary outcome) is measured using the dichotomised Glasgow Outcome Scale Extended (GOSE) conducted by telephone interview 6 months after injury. GOSE is dichotomised into 'unfavourable outcome' (GOSE 1-4), and 'favourable outcome' (GOSE 5-8). A medium-term functional outcome measure incorporating death and disability, rather than a short-term measure such as hospital-based mortality, was chosen. The quality of recovery (rather than just survival) after trauma is increasingly understood to be an important research outcome, as many injured patients who survive have long-term disability and are dependent on high levels of care. In addition, plasmin is known to affect immune system and neurological function as well as coagulation, and recent trials in TBI have found outcomes assessed at the time of hospital
Box 2 Primary and secondary outcome measures

\section{Primary outcome}

Dichotomised Glasgow Outcome Scale Extended (GOSE) at 6 months: the proportion of patients with a favourable outcome at 6 months (moderate disability or good recovery, GOSE scores 5-8), compared with those who have died (GOSE 1) or have severe disability (GOSE 2-4).

\section{Secondary outcomes}

1. Units of blood products used (packed red blood cells, fresh frozen plasma, platelets, prothrombin complex concentrate, recombinant factor Vlla, cryoprecipitate) in the first 24 hours.

2. Blood lactate concentration at patient arrival to hospital,

3. Coagulation profile (international normalised ratio, activated partial thromboplastin time, fibrinogen, platelet count) at:

a. hospital arrival;

b. end of treatment with study drug (ie, immediately after administering the second dose of the study drug by 8-hourinfusion);

c. 24 hours after the first dose of study drug.

4. Vascular occlusive events (deep venous thrombosis, pulmonary embolism, myocardial infarction, stroke) up until 28 days or hospital discharge (whichever occurs first).

5. Ventilator-free days in first 28 days.

6. Mortality at:
a. 24 hours;
b. 28 days;
c. 6 months.

7. Proportion of deaths due to:
a. bleeding;
b. vascular occlusion (pulmonary embolus, stroke or acute myocar- dial infarction);
c. multiorgan failure;
d. brain/neurological injury.

8. Cumulative incidence of sepsis up until 28 days or hospital discharge (whichever occurs first).

9. Quality of life (World Health Organisation Disability Assessment Schedule (WHODAS) 2.0 and EuroQol 5-Dimension (EQ-5D)) at 6 months.

discharge correlated poorly with long-term functional outcomes and, in at least one study, incorrectly predicted the direction of effect. ${ }^{35}$

Blood product usage (secondary outcome 1), ventilator-free days (secondary outcome 5) and quality of life measures (secondary outcome 9) are recorded on case report forms using the appropriate tools.

\section{Assessment for coagulopathy and acidemia (secondary outcomes 2 and 3 )}

Coagulation tests (international normalised ratio, activated partial thromboplastin time and fibrinogen levels) and full blood examinations including platelet counts are conducted for all patients as part of standard practice shortly after arrival to an emergency department. Laboratory analysis of venous blood lactate is also performed. Additional blood samples are collected at the end of the 8-hour infusion of the study drug, and 24 hours after the prehospital dose of study drug. 
Assessment for venous thromboembolism (secondary outcome 4)

To minimise the potential for selective outcome bias, all participants in a subgroup of centres undergo bilateral compression Doppler ultrasound between 5 and 7 days postinjury to examine for proximal lower limb deep venous thromboses (DVTs). In all centres, where there is clinical suspicion of DVT or pulmonary embolism $(\mathrm{PE})$, clinicians further investigate patients to confirm diagnosis. Results of any additional relevant diagnostic imaging are recorded. The incidence of DVT will be reported for the total study sample and in addition, among the subgroup of centres where routine Doppler ultrasound is protocolised.

\section{Assessment for cause of death (secondary outcome 7)}

In addition to all-cause death at 24 hours, 28 days and at 6 months (secondary outcome 6 ), among patients that die within 6 months the primary cause of death is categorised as: death due to bleeding; death due to vascular occlusion (including PE, stroke or acute myocardial infarction); death due to multiorgan failure that is not a direct result of bleeding or vascular occlusion; death due to brain and/or neurological injury or death due to another cause not classified. The principal investigator at each site is responsible for reporting the cause of death.

\section{Assessment for sepsis (secondary outcome 8)}

The cumulative incidence of sepsis will be collected up to 28 days or hospital discharge, whichever occurs first. Sepsis will be defined as: (1) clinical suspicion or confirmed infection $\geq 48$ hours after hospital admission; (2) at least two criteria for systemic inflammatory response syndrome and (3) commencement of antibiotics, or change to the current antibiotic regimen.

\section{Subgroup analyses}

The overall sample will be subgrouped by the following variables and the effect of the intervention assessed: age dichotomised to $\geq 50$ years; time from injury to first dose; first valid recorded systolic blood pressure categories $(\leq 75,76-89, \geq 90 \mathrm{~mm} \mathrm{Hg}$ ); mechanism of trauma (penetrating, blunt, burns) and baseline GCS $<9$.

\section{Statistical analysis}

The analysis and reporting of the results will follow the Consolidated Standards of Reporting Trials guidelines. ${ }^{37}$ Baseline characteristics will be tabulated by using appropriate summary statistics. Principal analysis of the primary outcome will be by intention-to-treat (ITT), including all randomised patients.

A modified ITT supporting analysis will also be presented that excludes patients who did not receive the study intervention after being randomised, or who were not eligible for randomisation. In addition, a perprotocol analysis will be presented for patients who satisfied all inclusion/exclusion criteria, received both doses of the study drug and who did not receive any open-label TXA (figure 2). All secondary end points will be analysed using the ITT population only. A nominal two-tailed 5\% significance level will be employed.

The primary outcome will be compared between treatment groups using a RR (95\% CI), and p value estimated by a log-binomial regression model. If model convergence is not achieved, then Poisson regression with robust SEs will be applied. Supplementary analyses will adjust for the randomisation stratification variables. If the proportion of patients missing the primary outcome exceeds $5 \%$, multiple imputation using chained equations will be employed using relevant baseline and postbaseline variables in the imputation models, constructed separately for each treatment arm. Post hoc adjustment for any variables exhibiting substantial imbalance across treatment arms at baseline will be performed and regarded as sensitivity analyses. Assessment of heterogeneity of treatment effect across prespecified subgroups will incorporate interaction term(s) in the regression models.

Binary secondary outcomes will also be analysed using log-binomial regression. Analysis of outcomes with approximately symmetric distributions will be analysed using linear regression to estimate the difference in means between treatment arms together with 95\% CIs and $\mathrm{p}$ values. Analysis of skewed outcomes and ventilator-free days will be analysed using quantile (median) regression, reporting the difference in medians between treatment arms together with 95\% CIs and $\mathrm{p}$ values. Additional analyses of binary outcomes at 28 days will be performed to take into account the competing risk of death using cumulative incidence functions, and similarly for analyses of causes of death at 6 months. For analysis of quality of life outcomes at 6 months, a value of 0 will be imputed for the EuroQol 5-Dimension (EQ5D) summary and VAS score for patients not alive at 6 months $^{38}$ and for World Health Organisation Disability Assessment Schedule (WHODAS), in the absence of published guidelines for addressing mortality a score of 61 will be imputed, placing death as worse than the maximum scale score of 60. Supplementary analyses of these quality of life end points will use inverse probability of death weighting rather than imputation of values to accommodate truncation by death. A detailed statistical analysis plan will be finalised prior to locking of the trial database and unblinding of treatment codes, and will be posted on the PATCH-Trauma study website.

\section{Sample size}

Targeting $90 \%$ power to detect an increase in a favourable GOSE outcome (scored 5-8) from $60 \%$ to $69 \%$ with TXA, this study would require 592 patients in each arm (1184 total) with a two-sided 5\% significance level. In a protocol amendment (PATCH Protocol ANZIC-RC/V.1.6 3 February 2020) accommodation for a $10 \%$ loss to follow-up, the required sample size was increased to 658 patients in each arm (1316 total).

This sample size is based on a conservative interpretation of results of the CRASH-2 study, in which the early mortality reduction was $13 \%$ and reduction of death due 


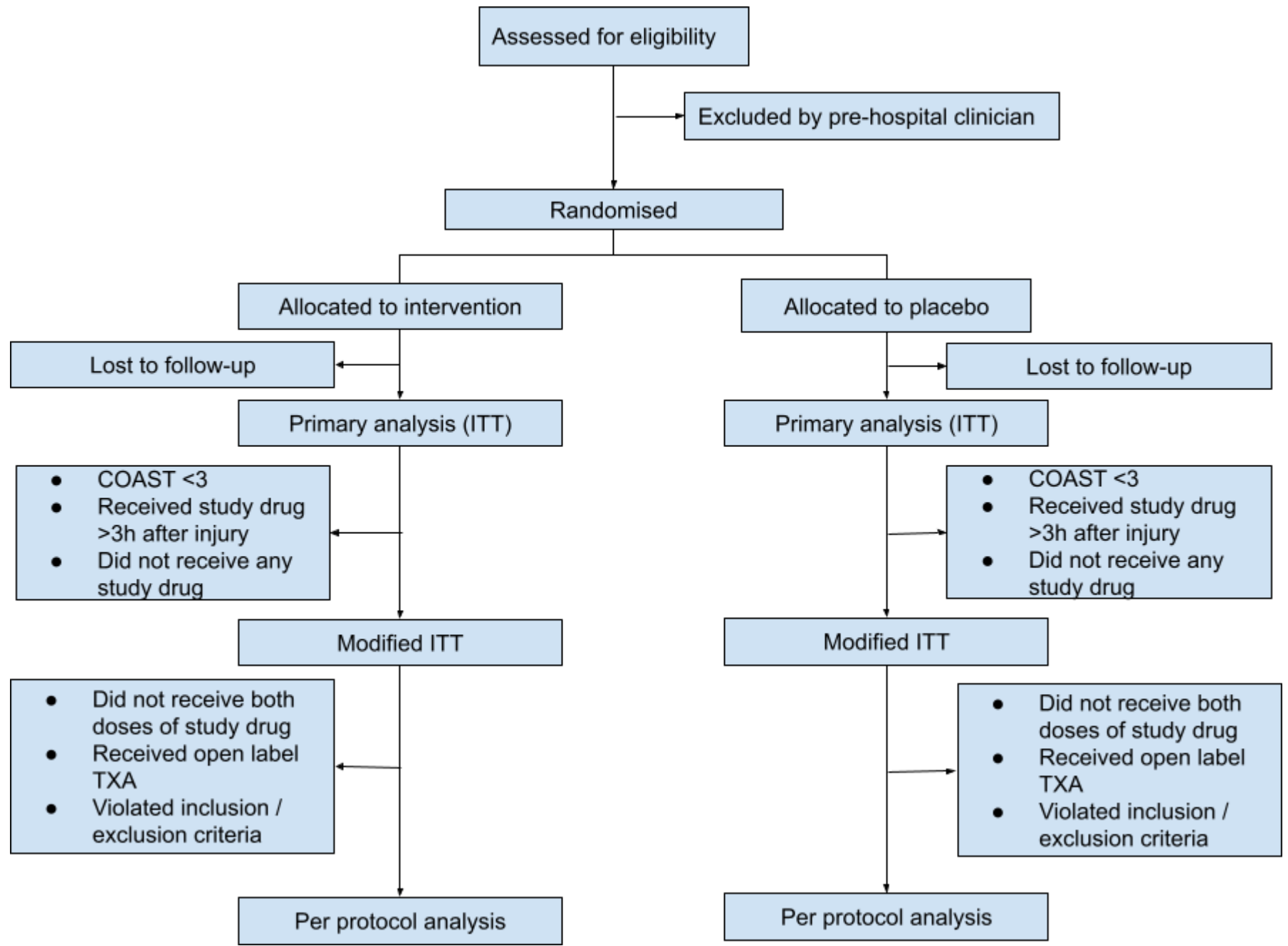

Figure 2 Analysis plan. COAST, Coagulopathy of Severe Trauma; ITT, intention-to-treat; TXA, tranexamic acid.

to bleeding was $32 \%-39 \%$. The PATCH study could be expected to observe a similar or greater effect of TXA because (1) it is enrolling only patients likely to be bleeding and coagulopathic and (2) the intervention is within 1 hour, and often $<30$ min of injury. Because patients in PATCH are bleeding and coagulopathic (factors strongly associated with early haemorrhagic deaths and late deaths due to single-organ or multiorgan failure) and because patients with isolated head injury are excluded by COAST $\geq 3$, the relevant end point from CRASH-2 to guide the expected effect size is the effect on death due to bleeding rather than all-cause mortality.

On the other hand, the observed effect of TXA in the PATCH study might be reduced compared to the restuls of the CRASH-2 trial because (1) given that high velocity blunt injury mechanism is responsible for most major trauma in Australia and New Zealand, some included multiply injured patients will have unsurvivable brain injury and (2) hospital-based clinicians will be aware that patients were enrolled in the study, and may know that a high proportion of enrolled patients will have ATC. With that knowledge, hospital clinicians may adjust their management to improve the underlying coagulopathy, hence the death/disability rate in the control group may be less than currently estimated $(54 \%)$.

\section{Data and safety monitoring, and interim analyses}

Two planned interim safety analyses for potential harm have been performed by the independent data and safety monitoring committee (DSMC) at 25\% and 50\% patient enrolment. Both analyses examined in-hospital and 28-day mortality using the Haybittle-Peto conventional 3-SD threshold of a standardised statistic (ie, $\left|Z_{k}\right|>3$ ) calculated from a normal approximation to the difference in mortality proportions. Based on the observed effects of the study drug and adherence to the study protocol in these analyses, the members of the DSMC were unanimous in recommending to the management committee continuation of the study to full enrolment.

\section{Patient and public involvement}

A patient representative $(\mathrm{AB})$ is part of the investigator group and provided input into the study design prior to first enrolment. 


\section{Ethics and dissemination}

This study is endorsed by the Australian and New Zealand Intensive Care Society Clinical Trials Group (ANZICS-CTG). The study is performed in accordance with the ethical principles of the Declaration of Helsinki (June 1964 and amended 1975, 1983, 1989, 1996, 2000, 2008 and Note of Clarification 2002 and 2004), ICH GCP Notes for Guidance on Good Clinical Practice (CPMP/ ICH/135/95) annotated with Therapeutic Goods Administration comments, the NHMRC National Statement on Ethical Conduct in Research Involving Humans (March 2007); the New Zealand Interim Good Clinical Research Practice Guidelines (Volume 21998 and Volume 3 2000) and ICH GCP Notes for Guidance on Good Clinical Practice (CPMP/ICH/135/95).

The study was approved by The Alfred Hospital Research and Ethics Committee project ID HREC/13/Alfred/9 (Local Reference: Project 214/13). The study is also approved in other Australian states and their respective ethics committees. Specifically, New South Wales (2019/ ETH00262), Queensland (HREC/14/QRBW/501), South Australia (490.14-HREC/15/SAC/14), Tasmania (Project ID 14471), Northern Territory (Reference ID 2016-1683). In New Zealand, Northern A Health and Disability Ethics Committee provided approval with project reference 14/NTA/123/AM11. In Germany, Witten/Herdecke University has provided ethics approval, project reference F-48/2020.

This study constitutes emergency research. Consistent with Principle 29 of the Declaration of Helsinki, patients who have suffered major trauma and are unable to provide informed consent are nonetheless entitled to participate in clinical research. In Australia, the National Health and Medical Research Council Statement makes provision for delayed and/or waiver of consent in time-critical interventions within the emergency or critical care setting. The study is performed in each centre where there is also a legal framework allowing for delayed and/or waiver of consent for research in emergency situations. Justifications for deferred/waived consent in this trial include the requirement for treatment to be administered as quickly as possible for maximum efficacy, and the perceived low risk of TXA.

Serious adverse events and suspected unexpected serious adverse reaction are reported within 24 hours of identification by telephone or email to the local principal investigator and the coordinating centre. However, consistent with the advice of Cook et al, adverse events already defined and reported as study outcomes (mortality, vascular occlusive events) will not be labelled and reported a second time as serious adverse events. ${ }^{39}$

\section{CONCLUSIONS}

Death from major trauma is common and disproportionately affects young adults. Early management with TXA has the potential to reduce haemorrhage and improve outcomes. The benefit of prehospital TXA in advanced trauma systems, when administered in conjunction with prehospital and in-hospital care that includes blood products, rapid angioembolisation and/or surgery, and early access to specialised critical care and rehabilitation, is currently uncertain. The PATCH-Trauma RCT aims to provide definitive guidance for clinicians on the utility of TXA during resuscitation after trauma.

\section{Author affiliations}

${ }^{1}$ Emergency and Trauma Centre, Alfred Hospital, Melbourne, Victoria, Australia

${ }^{2}$ National Trauma Research Institute, Melbourne, Victoria, Australia

${ }^{3}$ Department of Epidemiology and Preventive Medicine, Monash University School of Public Health and Preventive Medicine, Melbourne, Victoria, Australia

${ }^{4}$ Department of Intensive Care, Alfred Hospital, Melbourne, Victoria, Australia ${ }^{5}$ Ambulance Victoria, Melbourne, Victoria, Australia

${ }^{6}$ Australian and New Zealand Intensive Care Research Centre, Monash University, Melbourne, Victoria, Australia

${ }^{7}$ Greater Sydney Area Helicopter Emergency Medical Service, Sydney, New South Wales, Australia

${ }^{8}$ Sydney Medical School, Sydney University, Sydney, New South Wales, Australia ${ }^{9}$ Faculty of Medicine, The University of Queensland, Royal Brisbane and Women's Hospital, Herston, Queensland, Australia

${ }^{10}$ Joint Health Command, Australian Defence Force, Canberra, Australian Capital Territory, Australia

${ }^{11}$ Department of Intensive Care Medicine, Royal Brisbane and Women's Hospital, Brisbane, Queensland, Australia

${ }^{12}$ Critical Care Medicine, Auckland District Health Board, Auckland, New Zealand

${ }^{13}$ Cologne Merheim Medical Center, Department of Traumatology, Othopedic

Surgery and Sportsmedicine, University of Witten/Herdecke, Cologne, Germany

${ }^{14}$ Institute for Research in Operative Medicine, University Witten-Herdecke, Cologne, Germany

${ }^{15}$ College of Health and Medicine, Australian National University, Canberra, Australian Capital Territory, Australia

Twitter Biswadev Mitra @Biswadev_M

Collaborators PATCH-Trauma Study investigators: Russell Gruen, Stephen Bernard, Colin McArthur, Marc Maegele, Biswadev Mitra, Dashiell Gantner, Robert Medcalf, Michael Reade, Huyen Tran, Andrew Forbes, Paul S. Myles, Peter Cameron, Mark Fitzgerald, Stephen Rashford, Brian Burns, Tony Smith, Grant Christey, Zsolt J. Balogh, Anthony Trapani, Lynne Murray, Stefan Mazur, Camila Battistuzzo, Veronica Pitt, Ann-Marie Baker, Andy Swain, Paul Young, Jasmin Board, Nicole S. Ng, Sally Hurford.

Contributors RLG, BM and SB initiated the research and with AF and MCR, obtained initial funding. VP was the project coordinator and contributed to the design, initiation and patient recruitment in all sites. CMA is the project lead for New Zealand and responsible for initiation, enrolment and follow-up of the project in New Zealand. MM is the project lead in Germany and responsible for initiation, enrolment and follow-up of the project in Germany. DG, BB, LM and TT are members of the executive committee and have provided input into study design and execution. AF is also the chief biostatistician for the project. All authors have critically reviewed the manuscript for content.

Funding The study is funded by the Australian National Health and Medical Research Council (NHMRC APP1044894 and APP165275), the New Zealand Lottery Grants Board (34033) and by the Health Research Council of New Zealand (GA216F), and the German national research funding agency DFG (Deutsche Forschungsgemeinschaft MA 2569/6-1).

Competing interests None declared.

Patient consent for publication Not required.

Provenance and peer review Not commissioned; peer reviewed for ethical and funding approval prior to submission.

Open access This is an open access article distributed in accordance with the Creative Commons Attribution Non Commercial (CC BY-NC 4.0) license, which permits others to distribute, remix, adapt, build upon this work non-commercially, and license their derivative works on different terms, provided the original work is properly cited, appropriate credit is given, any changes made indicated, and the use is non-commercial. See: http://creativecommons.org/licenses/by-nc/4.0/. 


\section{ORCID iDs}

Biswadev Mitra http://orcid.org/0000-0002-0508-2450

Michael C Reade http://orcid.org/0000-0003-1570-0707

\section{REFERENCES}

1 Haagsma JA, Graetz N, Bolliger I, et al. The global burden of injury: incidence, mortality, disability-adjusted life years and time trends from the global burden of disease study 2013. Inj Prev 2016;22:3-18.

2 Dinh MM, Cornwall K, Bein KJ, et al. Health status and return to work in trauma patients at 3 and 6 months post-discharge: an Australian major trauma centre study. Eur J Trauma Emerg Surg 2016;42:483-90.

3 Beck B, Smith K, Mercier E, et al. Differences in the epidemiology of out-of-hospital and in-hospital trauma deaths. PLoS One 2019;14:e0217158.

4 Geeraedts LMG, Kaasjager HAH, van Vugt AB, et al. Exsanguination in trauma: a review of diagnostics and treatment options. Injury 2009;40:11-20.

5 Evans JA, van Wessem KJP, McDougall D, et al. Epidemiology of traumatic deaths: comprehensive population-based assessment. World J Surg 2010;34:158-63.

6 MacLeod JBA, Winkler AM, McCoy CC, et al. Early trauma induced coagulopathy (etic): prevalence across the injury spectrum. Injury 2014;45:910-5.

7 Mitra B, Cameron PA, Mori A, et al. Acute coagulopathy and early deaths post major trauma. Injury 2012;43:22-5.

8 Peltan ID, Vande Vusse LK, Maier RV, et al. An international normalized Ratio-Based definition of acute traumatic coagulopathy is associated with mortality, venous thromboembolism, and multiple organ failure after injury. Crit Care Med 2015;43:1429-38.

9 Draxler DF, Medcalf RL. Fibrinolysis and tranexamic acid: mechanistic principles. ANZ J Surg 2020;90:410-1.

10 Ker K, Edwards P, Perel P, et al. Effect of tranexamic acid on surgical bleeding: systematic review and cumulative meta-analysis. BMJ 2012;344:e3054.

11 CRASH-2 trial collaborators, Shakur H, Roberts I, et al. Effects of tranexamic acid on death, vascular occlusive events, and blood transfusion in trauma patients with significant haemorrhage (CRASH-2): a randomised, placebo-controlled trial. Lancet 2010;376:23-32.

12 CRASH-2 collaborators, Roberts I, Shakur H, et al. The importance of early treatment with tranexamic acid in bleeding trauma patients: an exploratory analysis of the CRASH-2 randomised controlled trial. Lancet 2011;377:1096-101.

13 Pusateri AE, Weiskopf RB, Bebarta V, et al. Tranexamic acid and trauma: current status and knowledge gaps with recommended research priorities. Shock 2013;39:121-6.

14 Brown JB, Neal MD, Guyette FX, et al. Design of the study of tranexamic acid during air medical prehospital transport (STAAMP) trial: addressing the knowledge gaps. Prehosp Emerg Care 2015;19:79-86.

15 Napolitano LM, Cohen MJ, Cotton BA, et al. Tranexamic acid in trauma: how should we use it? J Trauma Acute Care Surg 2013;74:1575-86

16 Valle EJ, Allen CJ, Van Haren RM, et al. Do all trauma patients benefit from tranexamic acid? J Trauma Acute Care Surg 2014;76:1373-8.

17 Cole E, Davenport R, Willett K, et al. Tranexamic acid use in severely injured civilian patients and the effects on outcomes: a prospective cohort study. Ann Surg 2015;261:390-4.
18 Moore HB, Moore EE, Gonzalez E, et al. Hyperfibrinolysis, physiologic fibrinolysis, and fibrinolysis shutdown: the spectrum of postinjury fibrinolysis and relevance to antifibrinolytic therapy. $J$ Trauma Acute Care Surg 2014;77:811-7.

19 Harvin JA, Peirce CA, Mims MM, et al. The impact of tranexamic acid on mortality in injured patients with hyperfibrinolysis. J Trauma Acute Care Surg 2015;78:905-11.

20 Binz S, McCollester J, Thomas S, et al. CRASH-2 study of tranexamic acid to treat bleeding in trauma patients: a controversy fueled by science and social media. J Blood Transfus 2015;2015:1-12.

21 Gruen RL, Jacobs IG, Reade MC. Tranexamic acid and trauma. Med J Aust 2014;200:255.

22 Roberts I, Edwards P, Prieto D, et al. Tranexamic acid in bleeding trauma patients: an exploration of benefits and harms. Trials 2017;18:48.

23 El-Menyar A, Sathian B, Asim M, et al. Efficacy of prehospital administration of tranexamic acid in trauma patients: a metaanalysis of the randomized controlled trials. Am J Emerg Med 2018;36:1079-87.

24 Nielsen K, Mock C, Joshipura M, et al. Assessment of the status of prehospital care in 13 low- and middle-income countries. Prehosp Emerg Care 2012;16:381-9.

25 Gruen RL, Mitra B. Tranexamic acid for trauma. Lancet 2011;377:1052-4.

26 Mitra B, Mazur S, Cameron PA, et al. Tranexamic acid for trauma: filling the 'GAP' in evidence. Emerg Med Australas 2014;26:194-7.

27 Perel P, Prieto-Merino D, Shakur H, et al. Predicting early death in patients with traumatic bleeding: development and validation of prognostic model. BMJ 2012;345:e5166.

28 Reade MC, Pitt V, Gruen RL. Tranexamic acid and trauma: current status and knowledge gaps with recommended research priorities. Shock 2013;40:160-1.

29 Shuster R, Mathew J, Olaussen A, et al. Variables associated with pulmonary thromboembolism in injured patients: a systematic review. Injury 2018;49:1-7.

30 Johnston LR, Rodriguez CJ, Elster EA, et al. Evaluation of military use of tranexamic acid and associated thromboembolic events. JAMA Surg 2018;153:169-75.

31 Morrison JJ, Dubose JJ, Rasmussen TE, et al. Military application of tranexamic acid in trauma emergency resuscitation (matters) study. Arch Surg 2012;147:113-9.

32 Lecker I, Wang D-S, Whissell PD, et al. Tranexamic acid-associated seizures: causes and treatment. Ann Neurol 2016;79:18-26.

33 Draxler DF, Medcalf RL. The fibrinolytic system-more than fibrinolysis? Transfus Med Rev 2015;29:102-9.

34 Mitra B, Cameron PA, Mori A, et al. Early prediction of acute traumatic coagulopathy. Resuscitation 2011;82:1208-13.

35 Thorn S, Tonglet M, Maegele M, et al. Validation of the coast score for predicting acute traumatic coagulopathy: a retrospective singlecentre cohort study. Trauma 2020;22:112-7.

36 Thorn S, Lefering R, Maegele M, et al. Early prediction of acute traumatic coagulopathy: a validation of the coast score using the German trauma registry. Eur J Trauma Emerg Surg 2019. doi:10.1007/s00068-019-01142-0. [Epub ahead of print: 29 Apr 2019].

37 Moher D, Hopewell S, Schulz KF, et al. Consort 2010 explanation and elaboration: updated guidelines for reporting parallel group randomised trials. BMJ 2010;340:c869.

38 Viney R, Norman R, King MT, et al. Time trade-off derived EQ-5D weights for Australia. Value Health 2011;14:928-36.

39 Cook D, Lauzier F, Rocha MG, et al. Serious adverse events in academic critical care research. CMAJ 2008;178:1181-4. 